\title{
The effect of McConnell taping on vastus medialis and lateralis activity during squatting in adults with patellofemoral pain syndrome
}

\author{
Sung-Eun Lee, Sung-Hyoun Cho* \\ Department of Physical Therapy, Daegu University, Gyeongsan, Korea
}

This study aims to examine the effect of McConnell taping to patients with patellofemoral pain syndrome on the change of the muscle activity of vastus medialis and vastus lateralis during squatting. The total numbers of participants are 16 patients with pain in their knee. There are three different experiments: no-taping, placebo taping, and McConnell taping. As a result, both the muscle activity of vastus medialis and muscle activity ratio of vastus medialis to vastus lateralis increased in placebo taping compared to no-taping, which wasn't statistically significant. However, the muscle activity of vastus medialis and muscle activi- ty ratio of vastus medialis to vastus lateralis increased in McConnell taping compared to no-taping, which was statistically significant. These results suggest that McConnell taping affects the activity of quadriceps by changing the position of the patella, and thus can effectively be applied in the treatment of patellofemoral pain syndrome.

Keywords: McConnell taping, Patellofemoral pain syndrome, Squatting, Quadriceps

\section{INTRODUCTION}

Patellofemoral pain is characterised by anterior or retropatellar pain associated with activities that load the patellofemoral joint, such as ascending or descending stairs, squatting, running and kneeling. Patellofemoral pain syndrome (PFPS) is the most common complaint affecting the knee (Wilson, 2007). 18.5-31\% in young adults complained of knee pain and $50 \%$ of the non-specific knee pain accounted PFPS (Rathleff et al., 2012). The factors causing PFPS include weakness and imbalance of Quadriceps muscle, malalignment of lower limb, stiffness of soft tissue, increase in Q-angle of quadriceps muscle, and overuse and abnormal movement of hip joint (Bolgla and Boling, 2011). Particularly if there is an imbalance of quadriceps muscle of thigh and a shortening of the lateral support attached to the lateral side of knee joint, patellar is an occurrence of tilting to the lateral side in the knee joint, which is a common symptom of PFPS (Lun et al., 2005).
PFPS has a risk of developing into chronic diseases such as chondromalacia or arthritis and so proper care and treatment is needed (Jeong and Kim, 2010).

Vastus medialis and vastus lateralis of quadriceps muscles are countervailing to adjust the location of knee joint and finally enhance the dynamic efficiency in knee function (Tang et al., 2001). Especially, vastus mediali has been suggested to act as a dynamic medial stabilizer (Gilleard et al., 1998; McConell, 1986), PFPS appear to decrease vastus medialis, that it may increase the lateral pull of the patella and reduce function at the knee joint (Baker et al, 2002; Chrisou, 2004). The patella malalignment changes the normal mechanism of knee extending, which brings about pathological changes of patellofemoral joint (Smith et al., 2009). To recover the mechanism of patellofemoral joint extending, locating an exact location of knee joint is important to increase the activity of vastus medialis and recover the start time, and more important to treat the patient with PFPS (Cowan et al., 2002). The conser-
${ }^{*}$ Corresponding author: Sung-Hyoun Cho

Department of Physical Therapy, Daegu University, 201 Daegudae-ro, Jinryang, Gyeongsan 712-714, Korea

Tel: +82-53-850-4350, Fax: +82-53-650-4350, E-mail: cory0831@naver.com

Received: April 1, 2013/ Revised: April 13, 2013/ Accepted: April 18, 2013
This is an Open Access article distributed under the terms of the Creative Commons Attribution Non-Commercial License (http://creativecommons.org/licenses/by-nc/3.0/) which permits unrestricted non-commercial use, distribution, and reproduction in any medium, provided the original work is properly cited. 
vative treatments of PFPS include taping to adjust the misalignment of lower limbs, stretching of soft tissue, strength of lower limbs, neuromuscular control training of quadriceps muscle of thigh, bio-feedback exercise (Aminaka and Gribble, 2008).

Corrective taping of the patella has since become a commonly used treatment for PFPS (Herrington, 2000). McConnell taping has devised a classification to describe abnormal patellar alignment. The four main malalignments include excessive lateral glide; excessive lateral tilt; excessive posterior tilt of the inferior pole and excessive rotation (McConnell, 1986, 1996). McConnell taping is designed to correct these malalignments and has four basic components, medial glide, medial tilt, anterior tilt and rotation. It is suggested that taping affects the tracking of the patella, facilitating its centralization within the trochlear groove ( $\mathrm{McC}$ Connell, 2002). If we look at the application of patellar taping, Christou (2004) applied patellar taping to normal subject and patient with PFPS and used isokinetic measuring instrument to see how active the muscle activity of vastus medialis and that of vastus lateralis is in terms of knee angle. As a result, the activity of vastus medialis increased, but that of vastus lateralis decreased in patients with PFPS. On the other hand, the activity of vastus medialis decreased, but that of vastus lateralis increased in normal person. Gilleard et al. (1998) investigated the effect of patellar taping on the timing of vastus medialis and vastus lateralis muscle activity in 14 female subjects with PFPS during stair ambulation with and without patellar taping. As a result, the onset of vastus medialis EMG activity compared with vastus lateralis occurred earlier in the knee movement with patellar taping than without taping during a step-up and step-down test. The authors discussed the possibility that patellar taping may enhance the onset of vastus medialis activity, which may result in improved patellar tracking (Gilleard et al., 1998). Many several studies showed that patellar taping is effective to reduce pain, increase the muscular strength of quadriceps muscle of thigh (Crossley et al., 2001), improve the neuromuscular mobilization, and correct the muscular contracture start time of vastus medialis against vastus lateralis (Cowan et al., 2002). However, most studies were restricted to examining pain or muscle activity of the quadriceps femoris muscle during simple actions and those concerning taping effects of the patella during functional motions including knee bending have been insufficient.

This study was to investigate the effects of muscle activity of quadriceps in the functional activity of squatting with and without McConnell taping.

\section{MATERIALS AND METHODS}

\section{Subjects}

The number of participants in this study is 16 patients $(8 \mathrm{Fe}-$ male, 8 Male) with knee anterior pain. The subjects of this study were patients with knee joint pain who visited $\mathrm{P}$ hospital located in Daegu Metropolitan City during October 2012. The subjects were limited to a person less than 80 points in funtional questionnaries (Kujala Patellofemoral Score) (Barby and Kevin, 2009).

The average age of subjects was $31.69 \pm 4.17 \mathrm{yr}$, average height was $167.51 \pm 8.24 \mathrm{~cm}$, and average weight was $61.82 \pm 10.90 \mathrm{~kg}$. After explaining about the purpose and content of this study prior to experiment to each and every participant, they are given a consent form. The requirements for experimental subjects are as follows: First, they should be less than 80 points in Kujala Patellofemoral Score. Second, they should be no history of hip joint, ankle, and foot joint dysfunction. Third, they should have no difficulty in squatting down. Fourth, they should have no orthopedic surgery history in lower extremity.

\section{Experimental procedure}

Sixteen participants squat in the following three conditions: no-taping, placebo taping with no tilting of knee joint, and patellar taping with medial tilting of knee joint. In no-taping, they squat with no taping attached. In placebo taping, they are taped at the same place as patellar taping without inducing the medial attraction of knee joint. In patellar taping, McConnell taping (McConnell, 1986) is applied, the knee joint is pushed toward the inside to induce medial attraction, and then they are taped before they squat. The advenced fixing tape (Endura-FIX, China) is attached to prevent the skin slipping and then nonelastic tape (Endura-FIX, China) is attached on it. A skilled physical therapist with more than 10 years of experiences helped to tape it.

When squatting down, their two foot angle is $120 \%$ on the basis of their shoulder (Earl et al., 2001), their two arms folded in the front, and trunk stood up to avoid lumbar flexion. For the knee angle, a semi-squatting posture is adopted as it is widely common to patients with PFPS: $50^{\circ}$ angle (Tang et al., 2001). Before exercise, $50^{\circ}$ is measured and then a bar is installed so that each participant could participate in the experiment at the same angle. For the order of exercise, the order is distributed randomly by drawing cuts. When doing each movement, they should keep for $5 \mathrm{sec}$ and rest for $30 \mathrm{sec}$, which is repeated three times. After exercise, tape is replaced by another one. And then they squat in another condition (Fig. 1). 


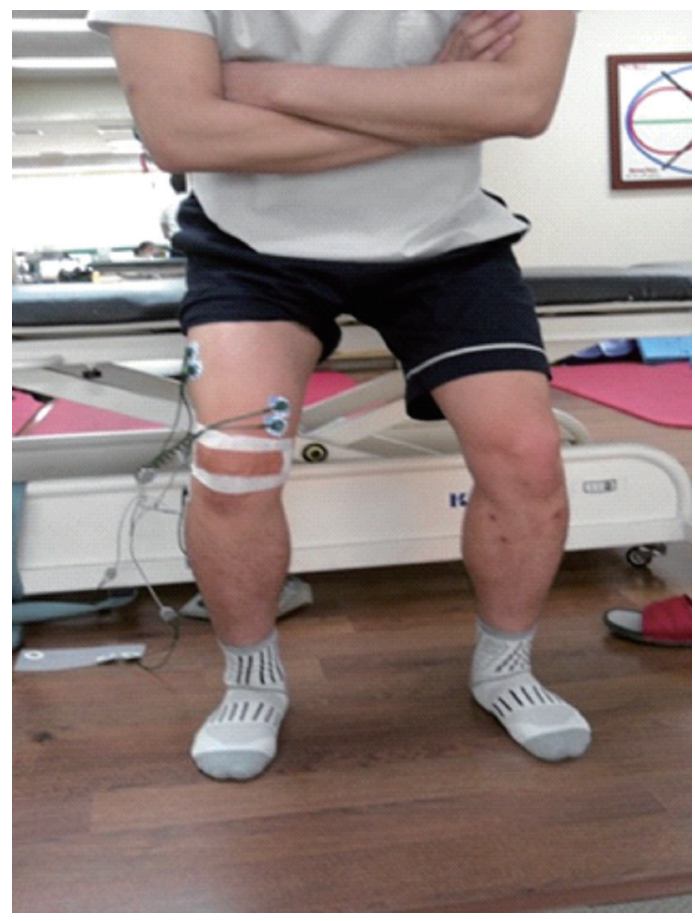

Fig. 1. EMG measurement during squatting.

\section{EMG and data processing}

For data collection, EMG Myosystem 1200 (Noraxon Inc., USA) System is used to measure the muscle activity of vastus medialis and vastus lateralis according to the taping. The attachment location of vastus medialis is $55^{\circ}$ in a vertical line from the upper and inner point of the knee joint: $4 \mathrm{~cm}$ upper and $3 \mathrm{~cm}$ inner from the knee joint. That of vastus lateralis is $15^{\circ}$ from the upper line of knee: $10 \mathrm{~cm}$ upper and 6-8 $\mathrm{cm}$ outer. The distance between electrodes is constant at $2 \mathrm{~cm}$ intervals. The single reference electrode is attached to knee joint. Before electrode attachment, skin is cleaned with an alcohol swab and shaved to reduce the skin resistance to less than $5 \mathrm{k} \Omega$ (Cowan et al., 2002). To obtain the maximum torque of each muscle, maximal voluntary isometric contraction (MVIC) of vastus medialis and vastus lateralis is obtained at $60^{\circ}$ knee joint in the squatting posture (Dixon and Howe, 2007). In the analysis of muscle activity, MVIC is measured with the root mean square (RMS) measured when contracting for $5 \mathrm{sec}$ from the knee muscle strength test (Kendall et al., 2005). After measuring each muscle activity in three different taping conditions, it is regarded as RMS and then each muscle activity for 3 sec in the middle is converted into a percentage (\% MVICRMS) as in the below. And the ratio between vastus medialis and vastus lateralis is calculated with normalized muscle activity value in each and different taping condition.

$$
\% \text { MVICRMS }=\frac{\text { RMS in each taping method }}{\begin{array}{c}
\text { RMS during the maximum } \\
\text { muscle contraction of knee joint }
\end{array}} \times 100
$$

\section{Data analysis}

All measures from this experiment are statistically treated by SPSS ver 12.0 (ICC, Chicago, USA). One-way repeated measured ANOVA is used to see if there is a difference between the measured muscle activity of vastus medialis and vastus lateralis and the ratio between the measured vastus medialis and vastus lateralis in three different conditions: no taping, patellar taping, and placebo taping. In post-test for the significant difference, pair-comparison is conducted with Bonferroni's correction. The significant level for statistical significance testing is at $\alpha=0.05$.

\section{RESULTS}

The average muscle activity of vastus medialis is $21.36 \pm 11.70 \%$ in no taping, $23.33 \pm 12.08 \%$, in McConnell taping, and 22.10 $\pm 10.64 \%$ in placebo taping. The average muscle activity of vastus lateralis is $19.02 \pm 9.08 \%$ in no taping, $18.08 \pm 7.34 \%$ in McConnell taping, and $18.92 \pm 8.96 \%$ in placebo taping. The activity ratio between vastus medialis and vastus lateralis is $1.14 \pm 0.40 \%$ in no taping, $1.29 \pm 0.42 \%$ in McConnell taping, and $1.20 \pm 0.36 \%$ in placebo taping.

In the comparison of the muscle activity between vastus medialis and vastus lateralis, the activity is significantly reduced in McConnell taping $(P<0.05)$. Also, in the comparison of the activity ratio between vastus medialis and vastus lateralis, the activity is significantly reduced in McConnell taping $(P<0.05)$. On the other hand, placebo taping is not statistically significant in the muscle activity between vastus medialis and vastus lateralis and the activity ratio between vastus medialis and vastus lateralis $(P>0.05)$ (Table 1) (Fig. 2).

\section{DISCUSSION}

The lateral tilting of patella is caused by the imbalance of vastus medialis and vastus lateralis. The shortening of the lateral support causes the relatively at weakness vastus medialis and so does not contribute to the dynamic medial stabilization (a major action of vastus medialis), thus resulting in abnormal movement of knee joint (McConnell, 2007). In McConnell taping, the use of inelastic tape is known to be effective to induce the medial attraction of lateral tilting knee joint, reduce pain with fixation, affect 
Table 1. The normalized EMG value for the vastus mealis and lateralis muscle (\% MVIC)

\begin{tabular}{lccc}
\hline \multirow{2}{*}{ Variables } & \multicolumn{3}{c}{ Taping condition } \\
\cline { 2 - 4 } & No taping & McConnell taping & placebo taping \\
\hline VMed & 21.36 & $23.33^{\text {a) }}$ & 22.10 \\
VLat & 19.02 & 18.08 & 18.92 \\
VMed/VLat ratio & $1.14: 1$ & $1.29:$ 1a) $^{\text {a) }}$ & $1.20: 1$ \\
\hline
\end{tabular}

Value: mean (SD), al $P<0.05$. VMed, vastus medialis activity; VLat, vastus lateralis activity; VMed/VLat, vastus medialis activity/vastus lateralis activity.

the functional activity, and change the start time of vastus medialis associated with vastus lateralis and reeducate the nerve root (Keet et al., 2007).

In this study, after McConnell taping to patellofemoral pain syndrom patients, they squat. The findings are that vastus medialis has the highest activity in placebo taping, followed by no-taping, McConnell taping and that the higher activity between vastus medialis and vastus lateralis indicates that McConnell taping may reduce the muscle activity; the values are significant. To see the similar previous studies to this founding, Evangelos and Christou (2004) applied McConnell taping to normal subject and patient with PFPS and used $30 \%$ s isokinetic leg presses measuring instrument to see how active the muscle activity of vastus medialis and vastus lateralis, pain. The medial patella taping procedure reduced preceived pain (70\%) and increased the activity of the vastus medialis and decreased the activity of vastus lateralis. In a study that examined maximal isometric muscle strength by applying patella taping, both concentric contraction and eccentric contraction increased (Herrington, 2001). According to the above studies, patella taping repositioned patellas in the trochlear groove, decreasing pressure in the patellofemoral joints, thereby effectively improving muscle strength of the quadriceps femoris muscle. And, McConnell was suggested, by using tape to correct patellar position, the vastus medialis is assisted in its efforts to resist the pull of the vastus lateralis and stabilize the patella (McConnell, 1996).

In this study, it is not statistically significant, but the muscle activity of vastus medialis and the activitiy ratio between vastus medialis and vastus lateralis increases numerically in placebo taping group compared to no-taping group. In a prior study, Christou (2004) noted that patella taping that was applied to patients with patellofemoral pain syndrome increased muscle activity of the vastus medialis and explained such change through decrease in pain of patellofemoral ligament and skin afferent stimulation. The result seems to be that taping promotes the tendon origin of vastus medialis and then afferent input is stimulated, and thus the

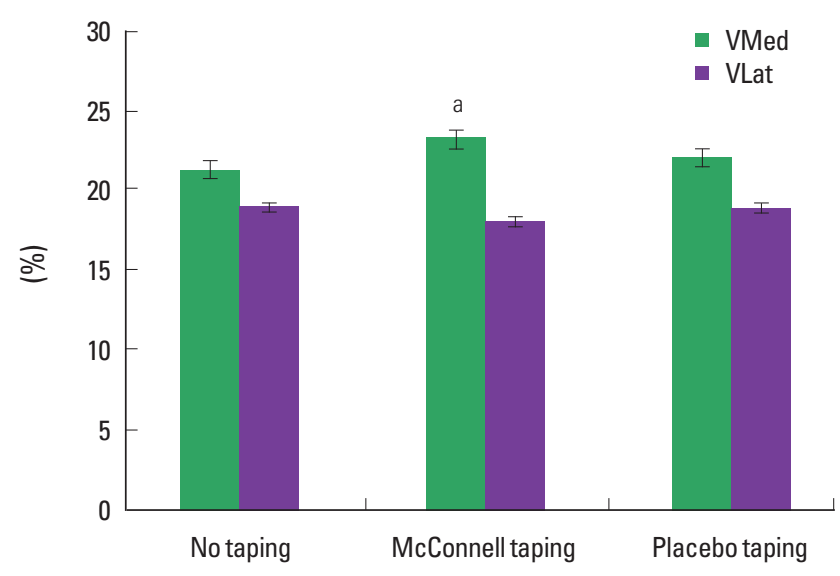

Fig. 2. The Normalized EMG data for vastus medialis (VMed), vastus lateralis (VLat) while performing three taping conditions. ${ }^{\text {al}} D$ ifferences detected by Bonferroni post-hoc analysis $(P<0.05)$

increase of stimulation of $a$-motor neuron source increases the muscle activity.

In the present study, a group to which McConnell taping was applied for medial drawing saw muscle activity of the vastus medialis and vastus medialis (VMO): vastus lateralis (VL) activation ratio increase and the reason may be explained as follows. Crossbridge formation by correcting the location of the patella and facilitation of muscle activity according to increase in skin afferent input is considered to have affected VMO activity.

This study is conducted in 16 adults in the late 20s and early 30 s, has problems of not considering age variable and limitations in generalizing the finding due to the limited number of subjects. And a study on correlation between changes in pain and changes in muscle activity is considered to be necessary. Also, participants couldn't take the same posture due to physical differences between individuals. More sophisticated equipment and optimal laboratory and apparatus are required and above problems should be supplemented.

\section{CONFLICT OF INTEREST}

No potential conflict of interest relevant to this article was reported.

\section{REFERENCES}

Aminaka N, Gribble PA. Patellar taping, patellofemoral pain syndrome, lower extremity kinematics, and dynamic postural control. J Athl Train 2008;43:21-28. 
Baker V, Bennell K, Stillman B, Cowan S, Crossley K. Abnormal knee joint position sense in individuals with PFPS. J Orthop Res 2002;20:208-214.

Barby, Kevin S. Anterior knee pain scale. Australian Journal of Physiotherapy 2009;55:140.

Bolgla LA, Boling MC. An update for the conservative management of patellofemoral pain syndrome: asystematic review of the literature 2000 to 2010. Int J Sports Phys Ther 2011;6:112-125.

Christou EA. Patellar taping increases vastus medialis oblique activity in the presence of patellofemoral pain. J Electromyogr Kinesiol 2004;14: 495-504.

Cowan SM, Bennell KL, Hodges PW. Therapeutic patellar taping changes the timing of vasti muscle activation in people with patellofemoral pain. Clin J Sport Med 2002;12:339-347.

Crossley K, Bennell KL, Green S, McConnell J. A systematic review of physical interventions for patellofemoral pain syndrome. Clin J Sports Med 2001;11:103-110.

Dixon J, Howe TE. Activation of vastus medialis oblique is not delayed in patients with osteoarthritis of the knee compared to asymptomatic participants during open kinetic chain activities. Man Ther 2007;12: 219-225.

Earl JE, Schmitz RJ, Arnold BL. Activation of the VMO and VL during dynamic mini-squat exercises with and without isometric hip adduction. J Electromyogr Kinesiol 2001;11:381-386.

Gilleard W, McConnell J, Parsons D. The effect of patellar taping on the onset of vastus medialis obliquus and vastus lateralis muscle activity in persons with patellofemoral pain. Phys Ther 1998;78:25-32.

Herrington L. The effect of patellofemoral joint taping. Crit Rev Physic Rehabil Med 2000;12:271-276.

Herrington L. The effect of patellar taping on quadriceps peak torque and perceived pain: a preliminary study. Physic Thera Sport 2001;2:23-28.

Jeong YH, Kim JI. Effects of a 9-week self-help management aquatic exercise program on pain, flexibility, balance, fatigue and self-efficacy in the patients with osteoarthritis. J Muscle Joint Health 2010;17:47-57.

Keet JHL, Gray J, Harley Y, Lambert MI. The effect of medial patellar taping on pain, strength and neuromuscular recruitment in subjects with and without patellofemoral pain. Physiotherapy 2007; 93:45-52.

Kendall FP, McCreary EK, Provance PE, Rodger MM, Romani WA. Muscles: Testing and function, with posture and pain. Philadelphia PA 2005; 212-213.

Lun VM, Wiley JP, Meeuwisse WH, Yanagawa TL. Effectiveness of patellar bracing for treatment of patellofemoral pain syndrome. Clin J Sport Med 2005;15:235-240.

McConnell J. Management of patellofemoral problems. Man Ther 1996;1: 60-66.

McConnell J. The management of chondromalacia patellae: a long term solution. Aust J Physiother 1986;32:215-222.

McConnell J. Rehabilitation and nonoperative treatment of patellar instability. Sport Med Arthrosc 2007;15:95-104.

McConnell J. The physical therapist's approach to patellofemoral disorders. Clin Sport Med 2002;21:363-387.

Rathleff MS, Roos EM, Olesen JL, Rasmussen S. Early intervention for adolescents with patellofemoral pain syndrome. BMC Musculoskelet Disord 2012;27:9.

Smith TO, Davies L, Donell ST. The reliability and validity of assessing medio-lateral patellar position: a systematic review. Man Ther 2009; 14:355-362.

Tang SF, Chen CK, Hsu R, Chou SW, Hong WH, Lew HL. Vastus medialis obliquus and vastus lateralis activity in open and closed kinetic chain exercise in patients with patellofemoral pain syndrome: an electomyographic study. Arch Phys Med Rehabil 2001;82:1441-1445.

Wilson T. The measurement of patellar alignment in patellofemoral pain syndrome: are we confusing assumptions with evidence? J Orthop Sport Phys Ther 2007;37:330-341. 